\title{
Stem Cells Era in Maxillofacial Surgery
}

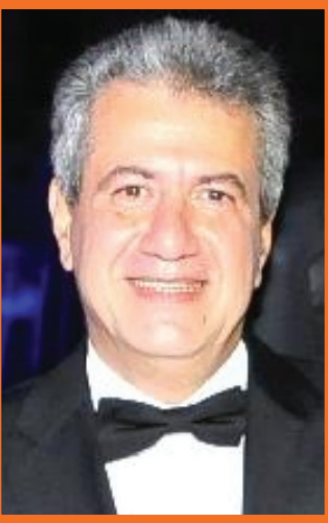

\section{Amr Bayoumi}

Professor of Oral and Maxillofacial surgery,Faculty of Dentistry, Alexandria University, Alexandria, Egypt and King Abdulaziz University, Jeddah, KSA.

Email: mobayme@kau.edu.sa
Prof. Amr Bayoumi is currently: - Professor and Consultant of Oral and Maxillofacial Surgery, Faculty of Dentistry, Alexandria University, Egypt, and King Abdulaziz University, KSA.

\section{Education:}

Bachelor of Dental Medicine and Surgery with a "very good" grade and an honors degree, Alexandria University (June 1983)

- Master's degree in Oral and Maxillofacial Surgery with a "very good" grade, Alexandria University (1988).

Ph.D. in Oral and Maxillofacial Surgery, Alexandria University (1991)

- Completed a course in Oral and Maxillofacial Radiology provided by the British government in King's College, London University, London, 1989 , and a clinical training course of Oral \& Maxillofacial surgery provided by Mount Vernon hospital, London, England.
Stem cells provide a new era for oral and maxillofacial surgery and reconstruction. Stem cells are colonlogic cells that have the ability to renew themselves and form various predecessors. They are found in common tissues used for wound healing and recovery after injuries. Large numbers of these cells are known to be instantly accessible; no immunological denial or rejection; do not have host disease against graft; Tumorigenesis do not occur; but there are some small ethical problems; The predictable differentiation potential for tissue separation and integration is not surprising.

There are two basic types of stem cells, depending on their origin: - Embryonic Stem Cells (ESCs): ESCs are initiated by a fetus that has been fertilized in an in vitro fertilization clinic $(100,200$ organized cell blastocysts). They are called a pluripotent cell population and are characterized by the ability to maintain the karyotype. ESCs can be divided into the three embryonic cotyledons (ectoderm, endoderm, and mesoderm); and in a culture; they form in colonies. These important highlights make ESCs affordable for future use in regenerative medicine and pharmaceuticals. Still; ethical discussions about the use of human embryos cannot be decided entirely; because in addition to scientific and practical questions, it is also dealt with through religious laws and philosophical reflections on the nature of human life.

- Adult stem cells: Adult stem cells are found in fetal and postnatal tissues; They are more specialized (multipotent) cells that participate in the repair and restoration of tissues, as well as in the maturation and aging processes. There are numerous sources of adult stem cells such as bone marrow (BMMSC); Adipose tissue (ATMSCs); Liver; Umbilical cord; and muscles. The intraoral sources are the dental pulp; The periodontal ligaments can also be a source of adult stem cells.

Fat and bone marrow stem cells are successful and are produced in the context of restorative work and aesthetics. However, ATMSCs have been less easily obtained without harmful effects on patients. Adipose tissue contains a population of mesenchymal stem cells that can be isolated and differentiated into various cell lines, including osteocytes; Fat cells; and myocytes depending on the culture conditions. These cells are called adipose derived stem cells (ASC).

The question arises here is: what do stem cells do? And the answer is so simple when you know that 10 million cells in your body die every minute every day and your own stem cells replace them so you can continue living.

Both undifferentiated ATMSC and BMMSC have osteogenic; chondrogenic and lipogenic differentiation potential. Both produce markers that help repair the deformity and have the same morphology; spindle shaped cells. Both appeared to work positively when used in reconstructive treatments. There are some contrasts between them; but the main imperative is; the ease of collection and the lower morbidity on the part of the donor. These two variables were extremely critical and favor the choice between fat and bone 
marrow for repair. ATMSCs and BMMSCs may be suitable replacements for the traditional intrusive method in the future. A better understanding of stem cell types, different platforms, and the influence of developmental components on the tissue design of different scaffolds, and how growth factors affect the tissue engineering pathway, opens opportunities to monitor recovery of bones and soft defects in the future, thus providing an alternative and innovative treatment for patients with soft / hard tissue defects.

Lectured in many national \& international scientific meetings.

Published 43 articles in peer reviewed and international journals. Member of the IADR.

Member of the Egyptian Society of Oral and Maxillofacial Surgery. Member of the Saudi Society of Oral and Maxillofacial Surgery.

Member of the Pan Arab Association of Oral and Maxillofacial Surgery. Member of the Egyptian Dental Syndicate.

Member of the Saudi Dental Society.

Member of the AOCMF Foundation.

Member of the editorial board of:

1-The journal of Oral and Maxillofacial surgery: clinics in Surgery.

2- The International journal of Dentistry and oral science.

3-The world journal of surgery and surgical research.

Reviewer for the International journal of Dentistry and Oral Health.

Reviewer for the Alexandria Dental Journal.

Reviewer for the Saudi medical Journal.

Reviewer for the Annals of Maxillofacial Surgery. 